\title{
Chromospheres of Luminous Cool Stars
}

\author{
A. K. Dupree \\ Harvard-Smithsonian Center for Astrophysics \\ 60 Garden St., Cambridge, MA 02138, USA \\ email: adupree@cfa.harvard.edu
}

\begin{abstract}
Direct ultraviolet imaging and spectroscopy of Alpha Orionis (Betelgeuse) reveals variable chromospheric structures and mass motions. Spectroscopy also demonstrates the changes of wind opacity, speeds, and mass loss in luminous stars. Cool stars have complex chromospheres that need to be considered in construction of stellar atmospheric models and subsequent spectral analyses.
\end{abstract}

Keywords. stars: atmospheres, stars: chromospheres, stars: imaging, stars: massloss, stars: supergiants

\section{Introduction}

Direct imaging and spectroscopy reveal that luminous cool stars - giants and supergiants - undergo changes in chromospheric structure including bright spots, wind variations, and changes in mass loss rates. Several bright objects have been intensively studied to demonstrate the complex time variable nature of their outer atmospheres. We are just beginning to address these complexities in the analysis of their spectra. Alpha Orionis (Betelgeuse) is perhaps the poster child of supergiant stars, and a brief summary of its behavior follows. However it is not alone among luminous stars, for many, if not most luminous giant stars also display chromospheres, varying atmospheric structure and mass loss. Atmospheric models and analysis techniques need to accommodate current observations.

\section{Betelgeuse}

This M-type supergiant has been the target of many studies, and merits special attention since it is the first and only star other than the Sun whose surface has been directly imaged (Gilliland \& Dupree 1996). Time sequences of ultraviolet images spanning $\sim 5$ years reveal the appearance of a few bright 'spots' that come and go on the chromospheric surface (Fig. 1). Schwarszchild (1975) estimated that there may be a few hundred convective cells on the surface of a red giant or supergiant, but no one was prepared for the appearance of only two or three. The frequency of imaging is not high enough to relate the appearance of bright regions to the $\sim 420$-day traveling waves detected in spatially unresolved ultraviolet spectra (Dupree et al. 1987) but it is reasonable to suspect that a connection is present. The 420-day period is believed to result from stellar pulsations and similar variations in radio flux have also been detected at $\mathrm{cm}$ wavelengths (O'Gorman, E. et al. 2015). Since chromospheric lines in Betelgeuse signal an outflow, an acceleration mechanism must be present in this warm region. Detection of a weak longitudinal magnetic field (Auriére et al. 2010) offers the possibility of Alfvén wave acceleration and heating. Recent models based on near-IR interferometric studies of the extended molecular layers of luminous stars (Arroyo-Torres et al. 2015) suggest that an acceleration mechanism in addition to pulsation and/or convective motions may be 


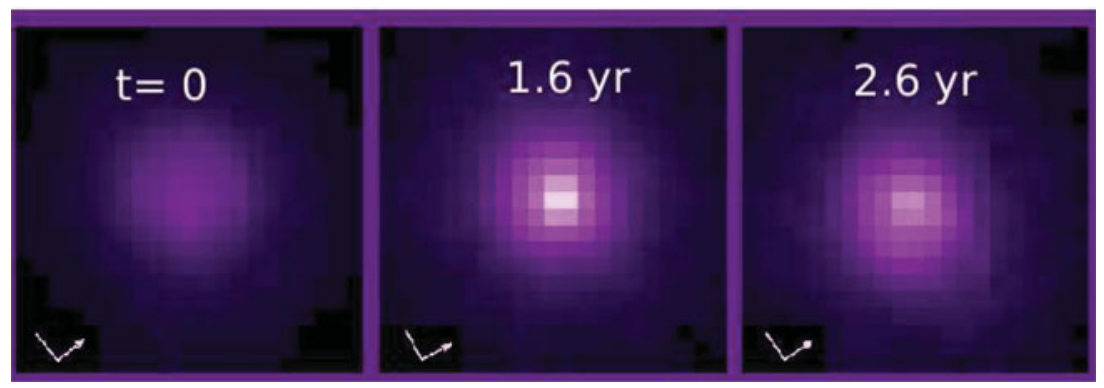

Figure 1. Near ultraviolet images of Alpha Ori (Betelgeuse) taken with HST/FOC with the same exposure time. The image contains $\sim 25$ resolution elements across the chromospheric disk (Dupree \& Stefanik 2013). Clearly substantial changes occur in the structure of the atmosphere.
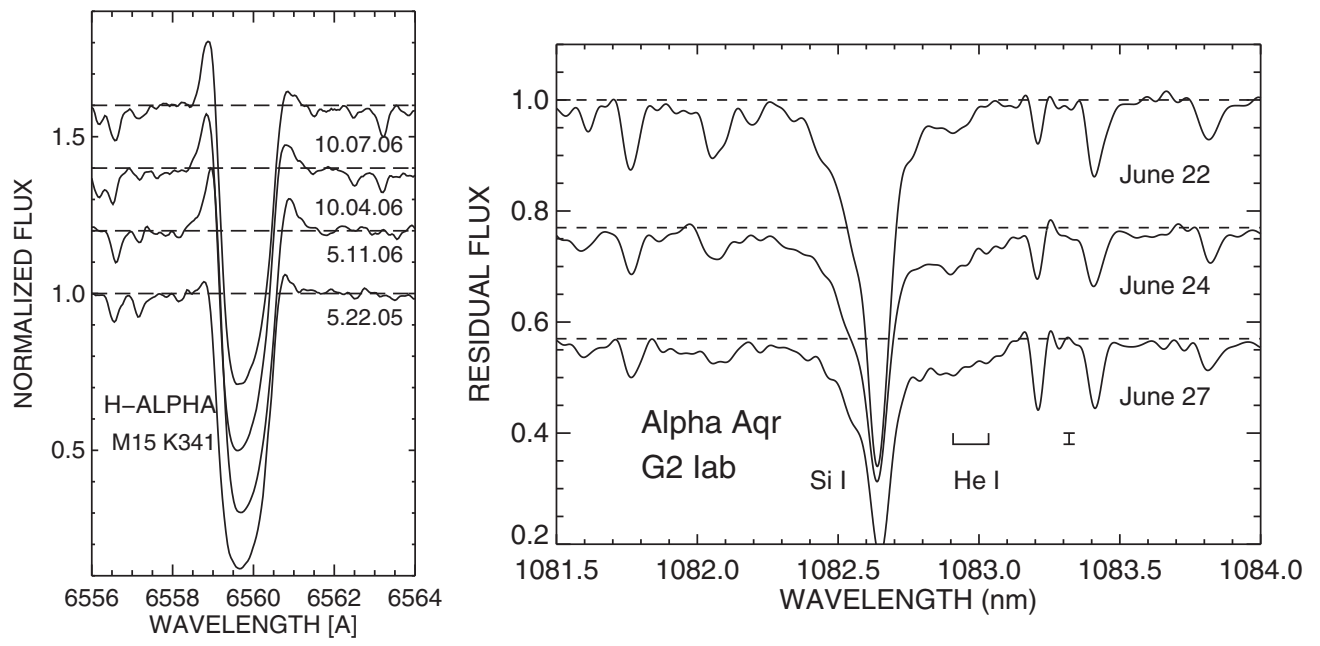

Figure 2. Left: H- $\alpha$ line from a metal-poor giant (K341) in the globular cluster M15, illustrating the change in emission wings; Right: The He I, $1083.0 \mathrm{~nm}$ near-IR transition in the supergiant $\alpha$ Aqr (HD 209750), displaying short term changes in the wind opacity and speed.

necessary - perhaps a contribution from radiative acceleration on molecular lines. Spectroscopic studies of Betelgeuse, again with spatial resolution across the surface, revealed (Uitenbroek et al. 1998, Lobel \& Dupree 2001) the orientation of the star, and detected substantial asymmetric motions throughout the chromosphere. There is no measurement of the $\sim 400$-day variation of the mass loss rate from Betelgeuse, however the impressive imaging of the circumstellar envelope and bow shock by the Herschel satellite (Decin et al. 2012) suggests that a 'clumpy' mass loss process is needed to understand the morphology of the circumstellar environment.

\section{Chromospheric Signatures}

Signatures of chromospheres are prevalent in spectra of all luminous stars. This is evident in optical spectra of metal-poor giants, such as H- $\alpha$ (Fig. 2) where emission frequently appears in the line wings. Even without emission, the profile can not be replicated with a radiative-convective model atmosphere. $\mathrm{H}-\alpha$ line cores are either shifted to shorter wavelengths or appear at the stellar radial velocity. Wing emission can change in asymmetry and strength signaling material flows towards or away from the star in the deep chromospheric layers. However the line cores, formed in the high chromosphere do not indicate inward-flowing material (Mészáros et al. 2009b). Analysis of typical profiles 
with complete non-LTE formulation and using appropriate semi-empirical chromospheric models shows that mass loss rate can vary over the sampling time-scale of $\approx 1$ year by factors of 1.5 to 6 in giant stars (Mészáros et al. 2009a).

Another good diagnostic of chromospheres and winds from warm $(\mathrm{T} \gtrsim 4500 \mathrm{~K})$ luminous stars lies in the near-IR He I line at $1083.0 \mathrm{~nm}$. Helium is formed at temperatures $\sim 15,000 \mathrm{~K}$, higher than stellar effective temperatures, and clearly signals the presence of a chromosphere. Because this transition arises from a metastable level, atoms will scatter the infrared continuum and the absorption profile maps out an extended expanding atmosphere. Such a profile can be seen in the spectrum of the supergiant $\alpha$ Aqr where absorption extends to high velocities $\left(\geqslant 90 \mathrm{~km} \mathrm{~s}^{-1}\right)$ and varies on a time scale of days (Fig. 2). At times, a P Cygni profile appears with emission from the extended chromosphere. Such observations also provide direct evidence of variable mass loss in supergiants and give confidence in the interpretation of the Herschel observations (Decin et al. 2012) of Betelgeuse mentioned earlier. While $\mathrm{H} \alpha$ and helium indicate temperatures of $\sim 15000 \mathrm{~K}$ or less, ions requiring temperatures up to $3 \times 10^{5} \mathrm{~K}$ have been detected in many giants and supergiants (Dupree et al. 2005) as well as X-ray emission (Ayres et al. 1995).

\section{Considerations for Atmospheric Models}

The ubiquitous nature and variability of chromospheres raises challenges for the interpretation of their spectra. Atmospheric models are advancing from radiative-convective equilibrium models to 2-D and even 3-D representations of a stellar photosphere. Given the absence of direct information about the surface structure and chromospheres of luminous stars, one wonders whether more complex models can give meaningful insight. Stellar abundances represent an additional problem. While LTE techniques are applicable for some transitions, the presence of non-LTE effects may enter to produce inaccuracies in abundance analyses. Careful study is needed of abundance determinations when models are constructed that better represent an actual stellar atmosphere.

\section{References}

Arroyo-Torres, B., Wittkowski, M., Chiavassa, A. et al. 2015, A\&A 575, A50

Auriére, M., Donati, J.-F., Konstantinova-Antonva, R., Perrin, G., Petit, P., \& Roudier, T. 2010, $A \& A 516, \mathrm{~L} 2$

Ayres, T. R., Fleming, T. A., Simon, T. et al. 1995, ApJS 96, 223

Decin, L., Cox, N. L. J., Royer, P. et al. 2012, A\&A A113

Dupree, A. K., Baliunas, S. L., Guinan, E. F., Hartmann, L., Nassiopoulos, G., \& Sonneborn, G. 1987, ApJ 317, L85

Dupree, A. K., Lobel, A., Young, P. R., Ake, T. B., Linsky, J. L., \& Redfield, S. 2005, ApJ 622, 629

Dupree, A. K. \& Stefanik, R. P. 2013, EAS Publications Series, ed. P. Kervella, T. LeBertre, \& G. Perrin, 60,77

Gilliland, R. \& Dupree, A. K. 1996, ApJ 463, L29

Lobel, A. \& Dupree, A. K. 2001, ApJ 558, 780

O'Gorman, E., Harper, G. M. Brown, A., Guinan, E. F., Richards, A. M. S., Vlemmings, W., \& Wasatonic, R. 2015, A\&A A101

Mészáros, Sz., Avrett, E. H., \& Dupree, A. K. 2009a, AJ 138, 615

Mèszáros, Sz., Dupree, A. K., \& Szalai, T. 2009b, AJ 137, 4282

Schwarzschild, M. 1975, ApJ 195, 137

Uitenbroek, H., Dupree, A. K., \& Gilliland, R. L. 1998, AJ 116, 2501 